http://dx.doi.org/10.35381/racii.v5i3.1105

\title{
Derechos Humanos desde el impacto ambiental de residuos sólidos
}

\section{Human Rights from the environmental impact of solid waste}

\author{
Alexandra Dolores Molina-Manzo \\ ub.alexandramolina@uniandes.edu.ec \\ Universidad Regional Autónoma de los Andes, Babahoyo \\ Ecuador \\ https://orcid.org/0000-0001-5015-9739 \\ Inés María Campuzano-Jarrín \\ db.inesmcj42@uniandes.edu.ec \\ Universidad Regional Autónoma de Los Andes, Babahoyo \\ Ecuador \\ July Dayanara Camino-Angulo \\ db.julydca25@uniandes.edu.ec \\ Universidad Regional Autónoma de Los Andes, Babahoyo \\ Ecuador
}

Recibido: 31 de octubre de 2020

Revisado: 10 de octubre de 2020

Aprobado: 05 de diciembre de 2020

Publicado: 10 de diciembre de 2020 


\title{
RESUMEN
}

La basura es el principal problema sanitario y ambiental mundo. En el cantón Montalvo, la disposición final de la basura, se encuentra ubicado en las riberas del rio Cristal y vertientes, no cuenta con ningún tipo de tratamiento, ocasionando terribles daños para la comunidad más cercana, que es el recinto la Ernestina. El objetivo general de la investigación es exponer la vulneración de derechos de los habitantes del recinto la Ernestina ya que están siendo afectados por la contaminación. La investigación es con una visión paradigmática cuantitativa, de tipo documental bibliográfica. Como métodos aplicados se utilizaron el sintético y el analítico. Se concluye que se hace necesario se respeten los derechos establecidos en la Carta fundamental permitiendo proteger la naturaleza y la salud de los habitantes, y se pueda dar cumplimiento con lo que reza en los postulados constitucionales respecto a los derechos de todas las personas y el medio ambiente.

Descriptores: Deterioro ambiental; derechos humanos colectivos; conservación de la naturaleza. (Palabras tomadas del Tesauro UNESCO).

\begin{abstract}
Garbage is the world's main health and environmental problem. In the Montalvo canton, the final disposal of the garbage is located on the banks of the Cristal river and its slopes, it does not have any type of treatment, causing terrible damage to the nearest community, which is the Ernestina enclosure. The general objective of the investigation is to expose the violation of the rights of the inhabitants of the Ernestina compound since they are being affected by the contamination. The research is with a quantitative paradigmatic vision, of a bibliographic documentary type. Synthetic and analytical methods were used as applied methods. It is concluded that it is necessary to respect the rights established in the Fundamental Charter, allowing the protection of nature and the health of the inhabitants, and to comply with what is stated in the constitutional postulates regarding the rights of all people and the environment. environment.
\end{abstract}

Descriptors: Environmental deterioration; collective human rights; nature conservation. (Words taken from the UNESCO Thesaurus). 
Iustitia Socialis. Revista Arbitrada de Ciencias Jurídicas.

Año V. Vol. V. №3. Edición Especial. 2020-III:

Universidad Regional Autónoma de los Andes

Hecho el depósito de Ley: FA2016000064

ISSN: 2542-3371

FUNDACIÓN KOINONIA (F.K). Santa Ana de Coro, Venezuela

Alexandra Dolores Molina-Manzo; Inés María Campuzano-Jarrín; July Dayanara Camino-Angulo

\section{INTRODUCCIÓN}

Los sitios de disposición final de residuos sólidos, que no fueron planeados técnicamente se conocen comúnmente como basureros a cielo abierto. Son terrenos en donde se depositan y acumulan los desechos urbanos municipales, sin ningún control técnico sanitario y operativo, así como la ausencia de obras de infraestructura para minimizar los impactos negativos al ambiente y su población.

Los vertederos al aire libre presentan graves problemas medioambientales a nivel global. Un tercio de todos los residuos urbanos generados en América Latina y el Caribe aún termina en basurales a cielo abierto, una práctica que está contaminando los suelos, agua y aire de la región, lo cual afecta la salud de sus habitantes, advirtió la Organización de la Naciones Unidas Medio Ambiente, en un informe regional presentado en Buenos Aires. "Cada latinoamericano genera un kilo de basura al día y la región en su conjunto, unas 541.000 toneladas". (ONU Medio Ambiente, 2018).

En el Ecuador, 109 de los 221 cantones tienen botaderos de basura a cielo abierto. Un total de 71 municipios cuentan con relleno sanitario y 41 con celdas emergentes, según los datos del Sistema de Información Ambiental del Ministerio del Ambiente Ecuador (MAE). De estos 109 que disponen la basura de forma incorrecta, 67 están en la Costa, 27 en la Sierra, 12 en la región Amazónica y 3 en la Insular. El 45,73\% de municipios refiere la presencia de recicladores informales, y se estima que el porcentaje total de reciclaje es de 14\%. A nivel nacional, la Gestión de Residuos Sólidos es competencia de los Gobiernos Autónomos Descentralizados (GADS) y el Ministerio del Ambiente (MAE) opera como instancia rectora. (Cuvi, 2015)

En el cantón Montalvo, el basurero a cielo abierto es un problema heredado de administraciones pasadas, empezando con la adquisición del terreno donde se encuentran toneladas de basura, en la cual no hicieron los estudios legales, ni técnicos ambientales para la ubicación del basurero, ni preguntaron a los moradores del sector, decisión tan importante en la que están vulnerando sus derechos. El 14 de febrero del año 2006, en sesión ordinaria del I. Concejo del Gobierno Municipal, deciden revocar la resolución de declaratorio de utilidad pública y ocupación inmediata, el terreno del Sr. Antonio Alarcón, y deciden autorizar el financiamiento y compra de un terreno de 
propiedad del señor Stalín Vargas, en un valor de $\$ 10,000$ cada cuadra. En Dicha acta de concejo, consta que el terreno pasó por una inspección por parte del municipio, y señalan no causar ningún impacto social; pero el municipio y sus autoridades omitieron, en la parte más fundamental que es el entorno natural, el río y las vertientes de agua, que en la actualidad se encuentran contaminadas, y el daño que causa a los habitantes del recinto la Ernestina. El señor Alarcón, presentó varios oficios a las administraciones anteriores, que se le reciba en sesión de concejo, para de manera conjunta resolver la situación del botadero de basura municipal, ya que él es colindante del mismo y afectado directo; pero dicha petición no tuvo el debido proceso para dar solución a esta crisis sanitaria.

Con todas estas quejas, elevó los reclamos al Ministerio del ambiente, y el 01 de abril del 2013 recibió respuesta de asesoría concerniente al cierre técnico del botadero de basura al cielo abierto del cantón Montalvo, dando un plazo al municipio de 30 días para elaborar un plan de contingencia para mitigar parcialmente los impactos detectados. EI MAE, determina que en el mismo lugar no es factible construir una celda emergente para la disposición final de los desechos sólidos.

Recomienda, que en el caso que no ubique dentro de su jurisdicción un lugar idóneo acorde lo dispone el Texto Unificado de Legislación Secundaria de Medio Ambiente (TULSMA), reactivar la mancomunidad de la Gestión GADS de Montalvo y Babahoyo, pero en la actualidad los dos cantones han crecido exponencialmente y las necesidades son diferentes. También señala una posible alternativa en la Mancomunidad Mundo verde, pero hasta la fecha el proyecto no avanza, dejando sin posibles soluciones al Municipio de Montalvo y con el paso del tiempo degenerando y contaminando los sectores aledaños y principalmente el río Cristal y sus vertientes.

Luego de revisar el registro de la gaceta municipal, en relación con la contaminación ambiental, solo se tiene la Ordenanza de determinación y recaudación de la tasa de recolección de desechos sólidos y aseo público. Sin embargo, no señala acerca de la disposición final adecuada que preserve el cuidado de la naturaliza y de sus habitantes. Lo que se puede presumir una ineficacia jurídica en la normativa del cantón Montalvo. La legislación ecuatoriana, en la Constitución de la República del Ecuador 
es pionera en el tema ambiental, en el derecho de la población a vivir en un ambiente sano, que garantice la sostenibilidad, se declara de interés público la preservación del ambiente, la conservación de los ecosistemas, la biodiversidad, la prevención del daño ambiental y la recuperación de los espacios naturales degradados. El Ecuador cuenta con diversos convenios, acuerdos o tratados internaciones acerca de la protección del medio ambiente como el Convención de las Naciones Unidas para el Cambio Climático, Protocolo de Kyoto, Convenio de Diversidad Biológica.

El Código orgánico organización territorial autonomía descentralizado (COOTAD), que señala en su artículo 136 comprende el ejercicio de las competencias de gestión ambiental, el ejercicio de la tutela estatal sobre el ambiente y la corresponsabilidad de la ciudadanía en su preservación. El artículo 443 del mismo cuerpo legal, manifiesto la gestión integral del manejo ambiental los Gobiernos Autónomos Descentralizados (GADS) de manera concurrente establecerán las normas para la gestión integral del ambiente y de los desechos contaminantes que comprende la prevención, control y sanción de actividades que afecten al mismo.

El Código orgánico del ambiente manifiesta respecto a la disposición final de los desechos sólidos urbanos lo siguiente: El derecho de las personas a vivir en un ambiente sano y ecológicamente equilibrado, así como proteger los derechos de la naturaleza para la realización del buen vivir (Art.1) Además de promover el efectivo goce de los derechos de la naturaleza y de las personas, comunas, comunidades, pueblos, nacionalidades y colectivos a vivir en un ambiente sano y ecológicamente equilibrado. (Art.4) (Ministerio del ambiente, 2018)

Por otro lado, el incumplimiento de este control conlleva acciones penales con delitos tipificados en el Código orgánico integral penal (COIP) capítulo cuarto delitos contra el ambiente y la naturaleza artículos 245, 246, 247, y también acciones civiles de demandas que pueden darse por la sociedad civil por medio del Código Orgánico General de Procesos (La Asamblea, 2015) en su artículo 38. Cabe también destacar que los municipios bajo las leyes tributarias tienen la capacidad de generar sus propias tasas e impuestos para la recolección y disposición final de desechos sólidos urbanos. 
En este sentido se manifiestan las preocupaciones por el ambiente, en concordancia con lo que manifiestan García-Batista, Socorro-Castro \& Maldonado (2019).

Dada la creciente preocupación de la sociedad por preservar el ambiente e implementar técnicas ecológicas que no afecten la salud de las personas y disminuyan el nivel de contaminación del aire, suelo y agua, que implica que se gestione y apliquen planes de manejo de los desechos y así contribuir a la protección del medio ambiente. (p.266)

En este contexto se puede observar que normativas existen para poder proteger adecuadamente la naturaleza. El Gobierno Autónomo Descentralizado (GAD) del cantón Montalvo, es el ente que tiene la competencia de manejar los desechos sólidos, (Art. 226- 231 COA) pero ante la despreocupación de las administraciones municipales, el Ministerio del Ambiente (MAE) notificó al Gobierno Autónomo Descentralizado, la aprobación técnica del estudio del cierre técnico y saneamiento del botadero de desechos sólidos. (Ministerio del ambiente, 2018)

Pues a pesar de toda la legislación que protege al ambiente y sanciones a los infractores, en la práctica las autoridades no han garantizado un medioambiente sano, pero también se requiere una ciudadanía activa que rechace este perverso modelo de basurero a cielo abierto y sea parte de la construcción de otras formas de vida saludables, solidarias y reparadoras del metabolismo social.

El objetivo general de la investigación es exponer la vulneración de derechos de los habitantes del recinto la Ernestina ya que están siendo afectados por la contaminación.

\section{METODOLOGÍA}

La investigación es con una visión paradigmática cuantitativa, de tipo documental bibliográfica. En virtud a la metodología que se utilizó en la presente indagación se encuentra organizada en un análisis específico, y recolección de información bibliográfica en función del inconveniente planteado, la contaminación ambiental ocasionada por el basurero y su repercusión en los habitantes del recinto La Ernestina, del Cantón Montalvo. Como métodos aplicados se utilizaron el sintético y el analítico. La población involucrada está conformada por los habitantes del recinto La Ernestina, 
cantón Montalvo compuesto de 200 personas, y de la cual se obtuvo una muestra de 67 habitantes. Se aplicó una encuesta conformada por cinco interrogantes y cuatro alternativas de respuesta.

\section{RESULTADOS}

Como se manifestó en la metodología aplicada en esta investigación, se procedió a realizar la siguiente encuesta a los involucrados en ella, que son los habitantes del recinto La Ernestina del cantón Montalvo, de acuerdo a la muestra extraída de la población, con el fin de obtener la información necesaria para la realización de la presente investigación.

1. ¿Cómo evaluaría la normativa que tiene el municipio para la protección o cuidado del medio ambiente en su recinto?

\section{Tabla 1.}

\begin{tabular}{|l|c|c|}
\hline RESPUESTA & FRECUENCIA & PORCENTAJE \\
\hline Buena & 0 & 0 \\
\hline Mala & 26 & $26,2 \%$ \\
\hline Muy buena & 0 & 0 \\
\hline Muy mala & 41 & $41,8 \%$ \\
\hline
\end{tabular}

Fuente: Datos suministrados de la encuesta aplicada a los habitantes del recinto La Ernestina. Elaboración propia (2020).

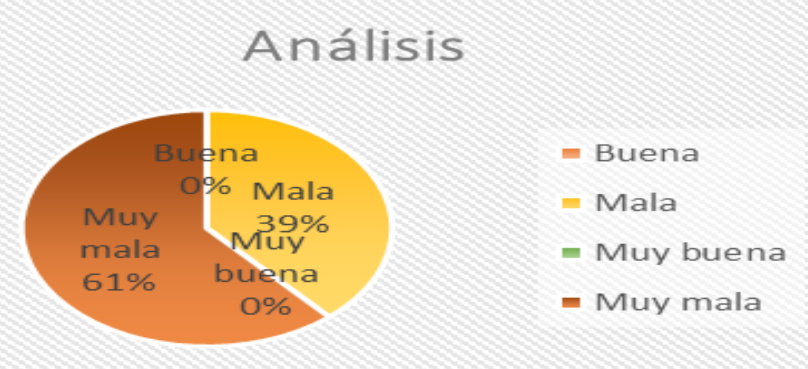

\section{Gráfica 1.}

Fuente: Datos suministrados de la encuesta aplicada a los habitantes del recinto La Ernestina. Elaboración propia (2020). 
2. ¿Cree usted que hay la gestión administrativa suficiente para gestionar la disposición final de los desechos sólidos?

\section{Tabla 2.}

\begin{tabular}{|l|c|c|}
\hline RESPUESTA & FRECUENCIA & PORCENTAJE \\
\hline No & 62 & $62,00 \%$ \\
\hline $\mathrm{Si}$ & 5 & $05.00 \%$ \\
\hline
\end{tabular}

Fuente: Datos suministrados de la encuesta aplicada a los habitantes del recinto La Ernestina. Elaboración propia (2020).

\section{Gráfico 2}

$$
\text { Si }
$$

No

\section{Gráfica 2.}

Fuente: Datos suministrados de la encuesta aplicada a los habitantes del recinto La Ernestina. Elaboración propia (2020).

3. ¿Quién cree usted que es el responsable de la contaminación ambiental?

\section{Tabla 3.}

\begin{tabular}{|l|c|c|}
\hline RESPUESTA & FRECUENCIA & PORCENTAJE \\
\hline EI GAD & $\mathbf{3 2}$ & $\mathbf{3 2 , 0 0 \%}$ \\
\hline $\begin{array}{l}\text { LoS } \\
\text { recicladores }\end{array}$ & $\mathbf{8}$ & $\mathbf{0 8 , 0 0 \%}$ \\
\hline La gente & $\mathbf{2 7}$ & $\mathbf{2 7 , 0 0 \%}$ \\
\hline
\end{tabular}

Fuente: Datos suministrados de la encuesta aplicada a los habitantes del recinto La Ernestina. Elaboración propia (2020). 


\section{Gráfica 3.}

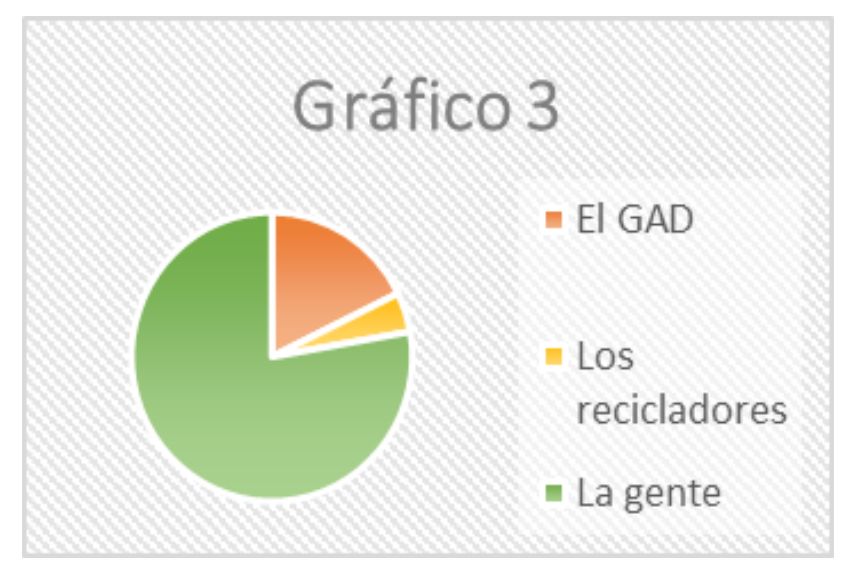

Fuente: Datos suministrados de la encuesta aplicada a los habitantes del recinto La Ernestina. Elaboración propia (2020).

4. ¿Cuál de los siguientes problemas ambientales es el que más afecta a su recinto?

\section{Tabla 4.}

\begin{tabular}{|l|c|c|}
\hline RESPUESTA & FRECUENCIA & PORCENTAJE \\
\hline Contaminación del aire & $\mathbf{7}$ & $\mathbf{0 0 , 7 \%}$ \\
\hline Contaminación del agua & $\mathbf{4 0}$ & $\mathbf{4 0 , 0 0 \%}$ \\
\hline Contaminación del suelo & $\mathbf{2 0}$ & $\mathbf{2 0 , 0 0 \%}$ \\
\hline $\begin{array}{l}\text { No existe problemas } \\
\text { ambientales }\end{array}$ & 0 & $\mathbf{0 , 0 0 \%}$ \\
\hline
\end{tabular}

Fuente: Datos suministrados de la encuesta aplicada a los habitantes del recinto La Ernestina. Elaboración propia (2020). 


\section{Gráfica 4.}

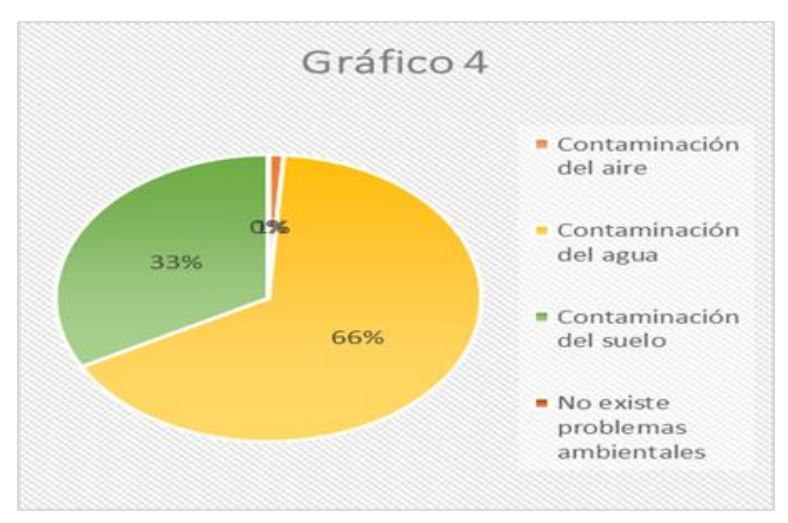

Fuente: Datos suministrados de la encuesta aplicada a los habitantes del recinto La Ernestina. Elaboración propia (2020).

\section{DISCUSIÓN}

Los resultados que se han obtenido en la siguiente investigación, queda en evidencia la falta de interés, preocupación por parte de las autoridades que, con el paso del tiempo, solo han ido pasando la crisis ambiental, a las siguientes administraciones de turno, sin dar una solución. Dejando la certeza que el basurero a cielo abierto es el resultado de irregularidades y falta de normas legales y técnica, vulnerando los derechos de sus ciudadanos y de la naturaleza. Se llegó a encontrar información de la forma en que fue adquirido el terreno y la falta de estudios legales y técnicos para la apertura de un basurero. Se entrevistó al señor colindante del terreno del basurero, y una encuesta a las personas del recinto la Ernestina, pues ellas están conscientes de la contaminación y que es competencia de la municipalidad dar solución y velar por los derechos fundamentales; como es vivir en un ambiente sano.

El Ministerio del ambiente, es conciso en los estudios que entregaron a la municipalidad en el año 2013, dando un plazo al municipio de 30 días para elaborar un plan de contingencia para mitigar parcialmente los impactos detectados. Además, determinó que en el mismo lugar no es factible construir una celda emergente para la 
disposición final de los desechos sólidos, dejando posibles alternativas de soluciones; pero que en la actualidad no son viables. Con el paso del tiempo degenerando y contaminando los sectores aledaños y principalmente el río Cristal y sus vertientes.

\section{CONCLUSIONES}

En esta investigación se ha expuesto conceptos, así como también los distintos aportes teóricos, consideraciones respecto al derecho ambiental y los derechos que tienen los ciudadanos en vivir en un espacio ecológicamente equilibrado, sus inicios, historia evolución, y el contenido establecido en el texto constitucional en el que claramente establece "El derecho de la población a vivir en un ambiente sano, que garantice la sostenibilidad, se declara de interés público la preservación del ambiente, la conservación de los ecosistemas, la biodiversidad, la prevención del daño ambiental y la recuperación de los espacios naturales degradados". De lo descrito se hace necesario que se respeten los derechos establecidos en la Carta fundamental permitiendo proteger la naturaleza y la salud de sus habitantes, para que se puedan dar cumplimiento con lo que reza en los postulados constitucionales respecto a los derechos de todas las personas y el medio ambiente. De esta apreciación y por cuanto nuestra propuesta persigue la elaboración de la ordenanza municipal para la creación de una empresa pública, con el objeto de intervenir en todas las fases de la gestión de residuos sólidos, bajo condiciones de preservación ambiental y precautelando la salud de los ciudadanos.

\section{FINANCIAMIENTO}

No monetario.

\section{AGRADECIMIENTO}

A la Universidad Regional Autónoma de Los Andes; por motivar el desarrollo de la Investigación. 


\section{REFERENCIAS CONSULTADAS}

Asamblea Nacional de la República del Ecuador. (2014). Código Orgánico Integral Penal. [Comprehensive Organic Criminal Code]. Recuperado de https://n9.cl/g6sc

Asamblea Nacional Constituyente de la República del Ecuador, (2008). Constitución de la República del Ecuador. Montecristi. Registro Oficial 449 de 20-oct-2008. Recuperado de https://n9.cl/sia

Asamblea Nacional de la República del Ecuador. (2015). Codigo Código Orgánico General de Procesos. [Code General Organic Code of Processes] Registro Oficial $N^{\circ} 506$.

Asamblea Nacional Constituyente. (2010). Código orgánico de organización territorial Autonomía y descentralización. [Organic code of territorial organization Autonomy and decentralization]. Registro Oficial Suplemento 303 de 19-oct2010 Recuperado de: https://n9.cl/d9vg

Asamblea Nacional (2010). Código Orgánico de Organización Territorial, Autonomía y Descentralización. [Organic Code of Territorial Organization, Autonomy and Decentralization]. Registro oficial 303 de 19 de oct-2010.

Asamblea Constituyente de la República del Ecuador. (2017). Código Orgánico del Ambiente.

Asamblea Constituyente. (2017). Código Orgánico del Ambiente. [Organic Environment Code]. Quito, Pichincha, Ecuador: Registro Oficial 983.

Cuvi, N. (2015). Ecología política y geográfica crítica de la basura en el Ecuador.[ Critical political and geographical ecology of litter in Ecuador]. Revista Latinoamerica de estudios socioambientales Facso Ecuador, 4-10. Recuperado de https://n9.cl/utluz

García-Bastida, R., Socorro-Castro, A. \& Vanessa-Maldonado, A. (2019) Manejo y gestión ambiental de los desechos sólidos, estudio de casos. [Control and environmental management of solid waste, case study]. Universidad $y$ Sociedad.11(1),265-271.

Ministerio del Ambiente (2018). Dirección Nacional de Control Ambiental. https://n9.cl/8xw76 


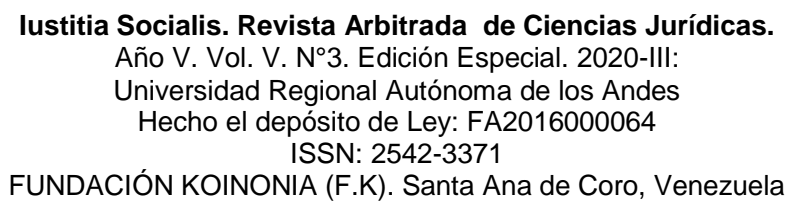

Alexandra Dolores Molina-Manzo; Inés María Campuzano-Jarrín; July Dayanara Camino-Angulo

Ministerio del Ambiente (2017). Texto Unificado de Legislación Secundaria de Medio Ambiente. [Unified Text of Secondary Environmental Legislation]. Decreto Ejecutivo 3516 Registro Oficial Edición Especial 2 de 31-mar-2003.

ONU Medio Ambiente. (2018). Cómo la basura afecta al desarrollo de América Latina. [How garbage affects the development of Latin America]. Recuperado de: https://n9.cl/7c3sj

(C2020 por los autores. Este artículo es de acceso abierto y distribuido según los términos y condiciones de la licencia Creative Commons Atribución-NoComercial-Compartirlgual 4.0 Internacional (CC BY-NC-SA 4.0) (https://creativecommons.org/licenses/by-nc-sa/4.0/). 